\title{
ANÁLISE DA FREQUÊNCIA DE INFECÇÃO DE FERIDA OPERATÓRIA DE ACORDO COM O TIPO DE ASSEPSIA E ANTISSEPSIA NO PRÉ-OPERATÓRIO
}

\author{
ANALYSIS OF THE FREQUENCY OF OPERATIVE WOUND INFECTION IN ACCORDANCE \\ WITH THE TYPE OF ASSEPSIA AND ANTISSEPSIA IN THE PRE-OPERATIVE
}

\author{
Israel Nunes Alecrin", Maria Elena Mandarim de Lacerda², Raiza Tinoco Manhães², Andreia Rodrigues \\ Campos $^{2}$, Giovanna Barreto de Oliveira Almeida ${ }^{2}$, Maria Alice Caldas Ferreira ${ }^{2}$, Juliana Siqueira Pessanha ${ }^{3}$. \\ 1. Doutor pela Universidade de São Paulo. Professor Titular da Cadeira de Ginecologia da Faculdade de \\ Medicina de Campos. Chefe do Serviço de Ginecologia do Hospital Escola Álvaro Alvim. \\ ${ }^{12}$ Discentes do Curso de Graduação em Medicina da Faculdade de Medicina de Campos. \\ ${ }^{3}$ Cardiologista. Professora da Faculdade de Medicina de Campos.
}

Resumo

Estudos sugerem que não há necessidade do uso de escovas para degermação pré-operatória das mãos, preconizando apenas o uso de antissépticos. A escovação parece aumentar as lesões nas mãos e disseminar bactérias; enquanto a utilização de antissépticos reduz o tempo de degermação e o uso de escovas. Objetivo: realizar uma análise comparativo entre a escovação tradicional pré-cirurgica das mãos com antissépticos e a degermação pré-cirurgica com a lavagem das mãos utilizando o antisséptico polivinilpirrolidona-iodo (PVP-I) ou clorexidine. Métodos: Para alcançar esses objetivos foi realizado um Estudo Documental e Exploratório de 200 cirurgias ginecológicas e de mastologia realizadas no Hospital Escola Alvaro Alvim, Campos dos Goytacazes, RJ. Os dados de interesse para o estudo foram obtidos a partir da coleta de dados de registro de cirurgias do centro cirúrgico e respectivo prontuário de acordo com a ficha clinica elaborada para a coleta das informações. As 200 cirurgias foram divididas em dois grupos sendo 100 delas realizadas por equipes que utilizaram a escovação das mãos (grupo A) e as outras 100 cirurgias à equipe apenas lavou as mãos com os antissépticos (grupo B) citados acima. Resultados: Foram observados quatro casos de infecção da ferida pós-operatória, dois no grupa $A$ e dois no grupo B. A análise dos resultados do presente estudo mostra que os dois métodos aplicados de assepsia précirúrgica, com e sem utilização de escovas, apresentaram eficiência e eficácia semelhantes, uma vez que não houve diferença estatística significante entre as taxas de infecção da ferida operatória.

Descritores: Lavagem das mãos, infecção da ferida operatória, cirurgia.

\begin{abstract}
Studies suggest that there is no need to use brushes for preoperative antisepsis of hands, only recommending the use of antiseptics. The brushing injury appears to increase the bacteria on the hands and spread, while the use of antiseptic antiseptic reduces the time and the use of brushes. Objective: The aim of this study was to conduct a comparative analysis between brushing traditional presurgical hand antisepsis with antiseptic and pre-surgically with handwashing using the antiseptic povidone-iodine (PVP-I) or chlorhexidine. Methods: To achieve these goals, a documentary and exploratory study of 200 gynecological surgeries and mastology performed in the University Hospital Alvaro Alvim, Campos dos Goytacazes, RJ. The data of interest for the study were obtained from the data collection record surgeries surgical center and its records according to established clinical record for the collection of information. The 200 surgeries were divided into two groups being 100 children conducted by teams that used the scrubbing hands (group A) and another 100 surgeries the team just washed his hands with antiseptic (group B) mentioned above. Results: Four cases of postoperative wound infection were observed, two in group $A$ and two in group $B$. The analysis of the results of the present study shows that the two applied pre-surgical asepsis methods, with and without the use of brushes, presented similar efficiency and effectiveness, since there was no statistically significant difference between the rates of infection of the surgical wound.

Keywords: Handwashing, wound infection, surgery.
\end{abstract}




\section{INTRODUÇÃO}

A infecção do sítio cirúrgico é uma frequente complicação que contribui com a elevação da morbidade e mortalidade dos pacientes pósoperados. Além disso, está relacionada com o aumento do tempo de internação, acarretando prejuízos físicos e emocionais ao paciente, bem como maiores custos com o tratamento (OLIVEIRA et al., 2007).

Os fatores de risco para infecção do sítio cirúrgico estão relacionados às condições de saúde do paciente, ao procedimento cirúrgico e ao grau de contaminação do local a ser operado (ROSENGREN et al., 2012). Entre aqueles inerentes ao paciente, estão: extremos de idade, obesidade, desnutrição, imunodepressão, tabagismo, diabetes mellitus, doença pulmonar obstrutiva crônica, alcoolismo, uso de corticosteroides e imunodepressores (MANGRAN et al., 1999). Já os que se relacionam ao procedimento cirúrgico, destacam-se as cirurgias de longa duração, a inadequação na administração da antibioticoprofilaxia e a presença de corpo estranho (MANGRAN et al., 1999).

Em relação ao grau de contaminação do local a ser operado, sabe-se que as cirurgias limpas além de não apresentarem processo infeccioso ou inflamatório, ocorrem em tecidos estéreis ou passíveis de descontaminação. O risco de infecção é menor que $5 \%$. Já as cirurgias potencialmente contaminadas também não apresentam processo infeccioso ou inflamatório, mas ocorrem em tecidos não passíveis de descontaminação ou em tecidos do trato digestivo, respiratório ou urinário. Apresentam risco de infecção próximo a $10 \%$. Enquanto as cirurgias contaminadas são aquelas realizadas em local de trauma recente e aberto, colonizado abundantemente por bactérias, apresentando risco de infecção entre 20 e $30 \%$. E por fim, as cirurgias infectadas são todas em que há tecido necrótico ou processo infeccioso no sítio cirúrgico, tendo maior risco de infecção que varia entre 30 e 40\% (CONCHAROGAZY et al., 2016).

Para diminuir o risco de infecção em ferida pós operatória, o antibiótico profilático deve ser administrado cerca de meia hora a uma hora antes da incisão e tem posologia máxima de 24 horas. Isso garante uma concentração inibitória mínima no início do procedimento, capaz de frear uma possivel contaminação durante a cirurgia (KARAMANIS et al., 2008; SANTOS et al., 2010). Essa conduta é recomendada em cirurgias potencialmente contaminadas ou contaminadas. Já em cirurgias limpas só está indicado quando são implantadas próteses ou em pacientes com fatores de risco, como obesidade, diabetes, imunossupressão, entre outros (MANGRAM et al.,1999; BELL, 2001; MALONE et al., 2002). Os procedimentos considerados sujos ou infectados merecem uso terapêutico, isto é, durante um período prolongado (KARAMANIS et al., 2008).

Outra conduta eficaz para diminuir a incidência de infecções da ferida operatória é a antissepsia pré-operatória das mãos e do sítio cirúrgico. Cabe ressaltar que antissepsia consiste na utilização de microbicidas sobre um determinado local com o objetivo de reduzir a quantidade de micro-organismos em sua superfície. Como exemplo desses produtos temos os alcoóis, a clorexidina, os compostos de iodo, iodóforos e triclosan. Enquanto assepsia é o conjunto de medidas propostas para impedir a introdução de agentes patogênicos em um meio estéril (MORIYA et al., 2008).

A higienização das mãos é descrita como uma medida individual simples que tem como objetivo diminuir a propagação das infecções hospitalares. Já em 1846 o médico húngaro Semmelweis relatou uma redução no número de mortes por infecção puerperal após a simples implementação da lavagem das mãos (DE COSTA, 2002). No entanto, a prática de higienizar as mãos com objetivo de evitar infecções hospitalares só se difundiu durante as últimas décadas (LOPES et al., 1999).

A microbiota das mãos constitui-se de bactérias residentes e bactérias transitórias. A flora residente, localizada nas camadas mais profundas da pele, é mais difícil de ser removida, porém não é comumente associada às infecções cruzadas. Nela, normalmente encontram-se as espécies de Corynecbacterium spp, Estreptococos coagulasse negativa e Micrococcus spp (GOULART et al., 2011). Já a flora transitória, adquirida pelo contato com os doentes ou com superfícies contaminadas, é a que coloniza a camada superior da pele, sendo mais facilmente removida pela lavagem das mãos. Esta flora inclui micro-organismos que são frequentemente associados à infecção nosocomial, incluindo Staphylococcus aureus, Enterococos e Bacilos Gram-negativos, como Pseudomonas spp, Klebsiella spp e Acinetobacter spp. (NOBLE et al., 1981; COUTO, 2001). Os vírus não são considerados 
parte da flora normal. São, portanto, incluídos como transitória ou como contaminação da flora e devem ser removidos durante as práticas de higienização das mãos (SATTAR et al., 2002).

De acordo com a nota técnica №01/2018 GVIMS/GGTES/ANVISA, a antissepsia pré-operatória das mãos pode ser realizada com o uso de esponjas para a realização da friç̧ão da pele com antisséptico degermante (Clorexidina $2 \%$ ou Polivinilpirrolidonaiodo - PVPI) ou por meio do uso de produto à base de álcool específico para fricção cirúrgica das mãos.

No entanto, estudos mostram que a prática tradicional de lavagem pré-cirúrgica das mãos, na qual se utiliza a escova, pode danificar a pele, resultando em: remoção das camadas da epiderme, aumento das contagens microbianas, alteração da flora microbiana, aparecimento de dermatites, abrasão da pele e aumento do risco de infecção (GALLE et al., 1978; MEERS e YEO, 1978; LOEB et al., 1997). Há evidências científicas sobre a segurança do uso de produtos a base de álcool, específico para antissepsia cirúrgica das mãos, podendo, portanto, substituir a técnica tradicional, com clorexidina ou polivinilpirrolidona iodo - PVPI degermante, no preparo pré-operatório das mãos (WHO, 2009; GONÇALVES et. al., 2012).

Cabe ressaltar que a eficácia do álcool depende de seu tipo, concentração e tempo de contato. Comparando a higienização das mãos por meio da utilização de escovas e por meio de soluções alcoólicas, tem-se verificado uma menor contagem bacteriana nas mãos quando se utilizam soluções à base de álcool, em particular, soluções formadas por $1 \%$ de gluconato de clorexidina e $61 \%$ a $70 \%$ de álcool (HOBSON et al., 1998; MULBERRY et al., 2001; ASSOCIATION OF SURGICAL TECHNOLOGISTIS, 2008).

As diretrizes mais recentes recomendam que os antissépticos cirúrgicos exibam um amplo espectro de atividade, ajam rapidamente e tenham um efeito persistente. A eliminação e redução da flora da pele transitória e residente, respectivamente, devem ocorrer imediatamente. A partir de então, deve se combater a contaminação por micro-organismos na mão enluvada.

A escassez de estudos sobre o tema e a persistência do hábito da escovação que continua como um mito ou dogma entre a maioria das equipes cirúrgicas e instituições fez despertar o interesse de verificar o impacto, em termos de eficácia, entre escovação pré-cirúrgica das mãos com antissépticos e a degermação pré-cirúrgica com a higienização das mãos utilizando antissépticos e sem escovas, nas taxas de infecção de ferida operatória.

O objetivo foi realizar uma análise comparativa das taxas de infecção de ferida operatória de pacientes submetidas a cirurgias ginecológicas e mamárias por equipes que utilizam a escovação pré-cirúrgica das mãos com antissépticos e equipes que utilizam apenas a degermação précirúrgica com a lavagem das mãos utilizando o antisséptico PVP-I ou clorexidane. Assim, esse estudo vislumbra contribuir com relevante ajuda no que tange a diminuição das taxas de infecção da ferida operatória de cirurgias ginecológicas e mamárias além de diminuir os gastos hospitalares com o uso de escovas e difundir esse conhecimento.

\section{MÉTODOS}

Foi realizado um estudo documental e exploratório de 200 cirurgias ginecológicas e mamárias realizadas no Hospital Escola Álvaro Alvim (HEAA), em Campos dos Goytacazes/RJ, com e sem a utilização de escovas para lavagem pré-cirúrgica das mãos. A maioria das cirurgias foi limpa ou potencialmente contaminada. Somente duas foram infectadas e não houve registro de cirurgias contaminadas

Foram incluídas mulheres de qualquer faixa etária. Entre os fatores considerados relevantes na análise estatística estão idade do paciente, profissão, cor, comorbidades, uso prévio de medicamentos, operações anteriores, realização prévia de cesárea, presença de anemia e câncer, motivo da cirurgia, tipo de cirurgia (limpa, infectada, contaminada), antibiótico profilaxia, infecção urinária no pósoperatório.

A coleta de dados foi feita pelo levantamento das notificações de infecções hospitalares nos prontuários decorrentes dos procedimentos cirúrgicos realizados, obtidos no $\mathrm{SClH}$ do HEAA. No processo de degermação das mãos foram utilizados dois tipos de antissépticos: o PVP-Ieoclorexidine. Uma equipe cirúrgica não utilizou a escovação, mas sim, a lavagem das mãos cuidadosamente, certificando-se de que as mãos e os espaços interdigitais tinham sido cobertos com os antissépticos, friccionando apenas a mão sobre toda a superfície de forma lenta e não traumaticamente, com a duração de 3 a 5 minutos. Tendo-se o cuidado 
de ter as unhas limpas e aparadas. Alem disso, tevese o cuidado de secar e "vestir" as luvas de forma correta. Os demais cuidados com os clientes foram idênticos para todas as equipes. Foi utilizada uma ficha de pesquisa elaborada pelo grupo pesquisador, onde foram registradas as informações coletadas.

Os dados foram tabulados com a ajuda do programa Epi Info 5.1. e analisados estatisticamente de acordo com o tipo de variável e o teste adequado para cada uma delas, em frequência de ocorrência de cada variável em estudo, a ser expressa em números absolutos.

A pesquisa foi condicionada ao cumprimento dos princípios éticos contidos na Declaração de Helsinki, da Associação Mundial Médica (http://www.wma.net/e/policy/17c_e.html), além do atendimento à legislação do
Comitê Nacional de Ética em Pesquisa. Foi submetida ao parecer do Comitê de Ética em Pesquisa (CEP) da Faculdade de Medicina de Campos dos Goytacazes/RJ, obtendo aprovação para ser realizada.

\section{RESULTADOS}

Foram coletados registros de 200 cirurgias, dentre as quais 100 cirurgias utilizaram a técnica com escovação e as outras 100 sem o uso das escovas. Das 200 cirurgias realizadas somente quatro apresentaram infecção do sítio cirúrgico, dentre as quais duas formam com a técnica com utilização de escovas e as outras duas sem a utilização de escovas. Em relação à faixa etária foi observado predomínio de pacientes de 51 a 60 anos, totalizando 61\%

Tabela 1 - Faixa etária

\begin{tabular}{lll}
\hline Faixa etária & Frequência & Percentual \\
\hline $\mathbf{1 8}$ a 30 & 9 & $4,50 \%$ \\
$\mathbf{3 1}$ a 40 & 32 & $16,00 \%$ \\
$\mathbf{5 1}$ a 60 & 122 & $61,00 \%$ \\
61 ou mais & 37 & $18,50 \%$ \\
\hline
\end{tabular}

Dos fatores de risco analisados $46 \%$ (86/200) dos pacientes apresentaram câncer, 14,5\% (29/200) anemia e 5\% (10/200) EAS com alterações no pré-operatório. Com relação ao percentual de comorbidades, $9 \%$ dos pacientes apresentam hipertensão arterial sistêmica e Diabetes Mellitus.

Dos fatores relacionados ao procedimento cirúrgico, 45,5\% (91/200) dos pacientes foram submetidos a cirurgias anteriores.

Quanto aos tipos de cirurgia podemos observar (Tabela 2), predomínio de cirurgias potencialmente contaminadas, totalizando $63,5 \%$ (127/200), seguidas de cirurgias limpas com 35,5\% $(71 / 200)$ e cirurgias infectadas representando $1 \%$ $(2 / 200)$ da amostra.

Tabela 2 - Tipos de cirurgia

\begin{tabular}{lll}
\hline Tipo de Cirurgia & Frequência & Percentual \\
\hline Limpa & 71 & $35,50 \%$ \\
Potencialmente contaminada & 127 & $63,50 \%$ \\
Infectada & 2 & $1 \%$ \\
\hline
\end{tabular}

Quanto a antibioticoprofilaxia durante a anestesia, $52,0 \%(104 / 200)$ das cirurgias realizadas foram utilizados cefazolina $1 \mathrm{~g} 2$ ampolas. Na antibioticoprofilaxia realizada no pós-operatório
49,5\% (99/200) das cirurgias foram utilizados cefazolina $1 \mathrm{~g}$ endovenosa de $8 / 8$ horas, como mostram as tabelas abaixo (Tabela 3 e 4 ) 
Tabela 3 - Antibioticoprofilaxia durante anestesia

\begin{tabular}{lll}
\hline Antibioticoprofilaxia durante anestesia & Frequência & Percentual \\
\hline Cefalotina 1g 1 ampola & 9 & $4,50 \%$ \\
\hline Cefalotina 1g 2 ampolas & 50 & $25,00 \%$ \\
Cefazolina 1g 1 ampola & 17 & $8,50 \%$ \\
Cefazolina 1g 2 ampolas & 104 & $52,0 \%$ \\
Cefazolina1g 3doses & 3 & $1,50 \%$ \\
Ciprofloxacino+ Metronidazol & 1 & $0,50 \%$ \\
Não & 14 & $7,00 \%$ \\
Não tinha ficha da anestesia & 2 & $1,00 \%$ \\
\hline
\end{tabular}

Tabela 4 - Antibioticoprofilaxia durante pós-operatório

\begin{tabular}{|c|c|c|}
\hline Antibioticoprofilaxia no pós-operatório & Frequência & Percentual \\
\hline Cefalexina 500mg 6/6h & 4 & $2,00 \%$ \\
\hline Cefalotina 1g EV 6/6h & 2 & $1,00 \%$ \\
\hline $\begin{array}{l}\text { Cefalotina 1g EV 6/6h + Cefazolina } 1 \mathrm{~g} \\
\text { EV dose única + Cefalexina 500mg 6/6h } \\
+ \text { Cefazolina 500mg 6/6h + Cefazolina } \\
\text { 500mg 6/6h }\end{array}$ & 1 & $0,50 \%$ \\
\hline Cefazolina 1g EV 4/4h & 4 & $2,00 \%$ \\
\hline Cefazolina 1g EV 6/6h & 7 & $3,50 \%$ \\
\hline $\begin{array}{l}\text { Cefazolina } 1 \mathrm{~g} \mathrm{EV} 6 / 6 \mathrm{~h} \text { e Cefalexina } \\
500 \mathrm{mg} 6 / 6 \mathrm{~h}\end{array}$ & 1 & $0,50 \%$ \\
\hline Cefazolina 1g EV 8/8h & 99 & $49,50 \%$ \\
\hline $\begin{array}{l}\text { Cefazolina 1g EV 8/8h + Cefalexina } \\
\text { 500mg 6/6h }\end{array}$ & 3 & $1,50 \%$ \\
\hline $\begin{array}{l}\text { Cefazolina } 1 \mathrm{~g} \mathrm{EV} \mathrm{8/8h}+\text { Cefazolina } \\
1 \mathrm{~g} \text { VO dose única }\end{array}$ & 2 & $1,00 \%$ \\
\hline $\begin{array}{l}\text { Cefazolina 1g EV 8/8h + Cefazolina } \\
\text { 500mg 12/12h }\end{array}$ & 1 & $0,50 \%$ \\
\hline Cefazolina $2 \mathrm{~g} \mathrm{EV}$ dose única & 4 & $2,00 \%$ \\
\hline Ciprofloxacino 200mg EV 12/12h & 1 & $0,50 \%$ \\
\hline Norfloxacino 400mg 1cp VO 12/12h & 1 & $0,50 \%$ \\
\hline Não & 70 & $35,00 \%$ \\
\hline
\end{tabular}


As complicações relatadas no pós-operatório podem ser analisadas através da tabela abaixo (Tabela 5).

Tabela 5 - Complicações no pós-operatório

\begin{tabular}{lll}
\hline Complicações & Frequência & Percentual \\
\hline Deiscência de sutura e seroma & 2 & $1,00 \%$ \\
\hline Hematoma & 1 & $0,50 \%$ \\
\hline Hematoma, Deiscência, ISC & 1 & $0,50 \%$ \\
\hline ISC & 2 & $1,00 \%$ \\
\hline Saída de secreção purulenta por via vaginal & 1 & $0,50 \%$ \\
\hline Seroma & 9 & $4,50 \%$ \\
\hline Seroma, ISC, Deiscência de FO & 1 & $0,50 \%$ \\
\hline Seroma e Hematoma de cúpula vaginal & 1 & $0,50 \%$ \\
\hline Não houve complicações & 182 & $91,00 \%$ \\
\hline
\end{tabular}

\section{DISCUSSÃO}

A análise comparativa entre as técnicas de antissepsia pré-operatória com escovas cirúrgicas e a lavagem das mãos utilizando PVP-I ou clorexidine não alterou os índices de infecção do sítio cirúrgico.

Resultados parecidos são encontrados na literatura quanto à comparação da eficácia da degermação cirúrgica das mãos com e sem o uso de escovas (GALLE e RHYNE, 1978; HIRAGA et al, 2006; LOEB et al. ,1997).

Vale ressaltar que os quatro casos de infecção do sítio cirúrgico, identificados neste estudo, ocorreram em paciente que apresentavam fatores de risco de maior relevância.

No grupo operado com a técnica de lavagem cirúrgica das mãos com a utilização de escovação, as duas pacientes com infecção da ferida operatória eram idosas e imunodeprimidas. Uma tinha ainda Diabetes Mellitus e foi submetida à segmentectomia lateral da mama associado à linfadenectomia axilar. A outra paciente tinha história de um parto cesáreo e foi submetida à quadrantectomia da mama associada à dissecção axilar. Ambas para tratamento de carcinoma ductal infiltrante da mama.

Quanto ao grupo operado com a técnica sem escovação, as duas pacientes com infecção da ferida operatória também eram idosas e imunodeprimidas. Uma delas ainda era obesa com história de dois partos cesáreos. Além disso, não foi utilizada antibioticoprofilaxia nestes dois casos, sendo que em um deles, uma cirurgia potencialmente contaminada (histerectomia abdominal total), estaria indicada. Estes dois casos também estavam associados a outras complicações da ferida operatória como hematoma, deiscência de ferida operatória, área de necrose no local da ferida e seroma.

Sabe-se que cirurgias prévias podem resultar em dificuldades técnicas no ato operatório, o que demanda maior tempo cirúrgico. Isso aumenta a incidência de complicações. A estimativa é que o risco de infecção dobre a cada hora de cirurgia (MANGRAM, 1999; FAHEL et al., 2001; MALLAGONI e GAGLIARD, 2005; COELHO, 2010).

Cabe relatar que três casos de infecção da ferida operatória foram identificados ainda durante a internação. Somente em uma paciente, a infecção do sítio cirúrgico foi diagnosticada após a alta hospitalar, durante o seguimento ambulatorial.

É importante destacar que a notificação da infecção cirúrgica, determinada apenas durante a permanência hospitalar não fornece dados 
fidedignos e subestima as verdadeiras taxas de infecção pós-cirúrgicas (OLIVEIRA et al., 2007).

Vários estudos mostraram que uma proporção importante de infecções pós-operatórias se desenvolvem após a alta, de modo que o a companhamento dos pacientes ambulatorialmente é uma estratégia muito importante quando adotada. (FERRAZ et al., 1992.; OLIVEIRA et al., 2007).

De acordo com vários autores, (BURNS e DIPPE, 1982; FERRAZ et al., 1992; OLIVEIRA et al., 2007), verifica-se a ocorrência de um grande número de infecções do sítio cirúrgico até o sétimo dia pós- cirurgia. Sendo assim, a alta precoce pode ser um problema para o diagnóstico das infecções que se manifestam nos primeiros dias de pós-operatório, o que ocorreu com a maioria das cirurgias presentes neste estudo, cuja permanência hospitalar após a cirurgia foi em sua maioria por três dias.

\section{CONCLUSÃO}

Os dois métodos aplicados de assepsia précirúrgica, com e sem utilização de escovas, apresentaram eficiência e eficácia semelhantes, uma vez que não houve diferença estatística significante entre as taxas de infecção da ferida operatória.

\section{REFERÊNCIAS}

ASSOCIATION OF SURGICAL TECHNOLOGISTIS. Recommended Standards of Practice for the Surgical Scrub.2008.

BELL, D.M. Promoting appropriate antimicrobial drug use: perspective from the Centers for Disease Control and Prevention. Clin Infect Dis., v.33, p. 245-50, 2001.

Brasil. Agência Nacional de Vigilância Sanitária. Nota técnica N01/2018 GVIMS/ GGTES/ANVISA: Orientações gerais para higiene das mãos em serviços de saúde.

BURNS, J.J; DIPPE, S.E. Postoperative wound infections detected during hospitalization and after discharge in a community hospital. Am J Infect Control.v.10,n.2,p. 60-5.1982.

COELHO, F.A. Avaliação da profilaxia antimicrobiana em hernioplastia a Linchtenstein. 2010. 50 p. Dissertação (Mestrado em Ciências da saúde) - Universidade Federal do Sergipe, Aracaju, 2010.

CONCHA-ROGAZY, M.; ANDRIGHETTI-FERRADA, C.; CURI-TUMA, M. Aseptic techniques for minor surgical procedures. Rev. méd. Chile, Santiago, v. 144, n. 8, p. 1038-1043, Aug. 2016.

COUTO, R.C. Infecção hospitalar. 1.ed. Rio de Janeiro: médica e Científica, 2001. Lavagem das mãos. p.121-131.

DE COSTA, C.M. 'The contagiousness of Childbed Fever': a short history of puerperal sepsis and its treatment. Med J Aust, v. 177.2002.

FAHEL, E.; AMARAL, P.; AZARO, E. Manual de atualização em cirurgia geral, Rio de Janeiro, Revinter: 2001.

FERRAZ, E.M; BACELAR, T.S; AGUIAR, L. Wound infection rates in clean surgery: a potencially mesleading risk classification. Infect Control Hosp Epidemiol. v.13, n.8, p.457-63. 1992.

GALLE, P.C; HOMESLEY, H.D; RHYNE, A.L. "Reassessment of the surgical scrub". Surgery,Gynecology \& Obstetrics , v.147, n. 2, p. 215218.1978.

GONÇALVES, K.J; GRAZIANO, K.U; KAWAGOE, J.Y. Revisão sistemática sobre antissepsia cirúrgica das mãos com preparação alcoólica em comparação aos produtos tradicionais. Rev Esc Enferm USP, v 46, n. 6, p. 1484-93, 2012.

GOULART, D.R.; ASSIS, E.A.; DE-SOUZA, M.T. Avaliação microbiológica da antissepsia pré-operatória das mãos. Rev. cir. traumatol. bucomaxilo-fac.[online]. 2011, vol.11, n.3, pp. 103-112. ISSN 1808-5210.

HIRAGA, W.M. et al. Escovação ou lavagem das mãos no centro cirúrgico: estudo comparativo. In: VI Congresso Pan-Americano, X Congresso Brasileiro de Controle de infecções e Epidemiologia Hospitalar, 2006. Porto Alegre.

HOBSON D.W. et al. Development and evaluation of a new alcohol-based surgical hand scrub formulation with persistent antimicrobial characteristics and brushless application. Am J Infect Control, v.26, n., oct. 1998.

KARAMANIS, E. et al. World Wide Web resources on prevention and treatment of postoperative infection. Am J Surg., v.196, p. 307-10, 2008.

LOEB M.B. et al. A randomized trial of surgical scrubbing with a brush compared to antiseptic soap alone. Am J Infect Control, v.25, n. 1 , feb. 1997.

LOPES, M.H.; MOROMIZATO, S. S.; VEIGA J. S.; Adesão às medidas de precaução-padrão: relato de experiência. Rev. Latino-Am.

Enfermagem, Ribeirão Preto, v. 7, n. 4, Oct. 1999.

MALLAGONI, M.A.; GAGLIARD, R.J. HERNIAS. In: Townsend CM, editor. Sabiston, Tratado de cirurgia 17o ed. Rio de Janeiro: Elsevier; 2005. p.1199-1218.

MANGRAM, A.J. et al. Guideline for Prevention of Surgical Site Infection. Hospital Infection Control Practices Advisory Committee. Infect Control Hosp Epidemiol.v.20, n4, p. 250. 1999.

MANGRAN, A.J. Guideline for prevention of surgical siteinfection. American Am J Infect Control., v.27,n.2,p.97-132, 1999.

MARTINS, M.A. Manual de Infecção Hospitalar. Epidemiologia, prevenção e controle. 2 ed.Rio de Janeiro: MEDSI, 2001,1116 p.

MEERS, P.D; YEO, G.A. "Shedding of bacteria and skin squames after handwashing". The Journal of Hygiene, Lond., v.81, n.1.1978. 
MORIYA, T; MÓDENA, J.L.P. Assepsia e antissepsia: técnicas de esterilização. 2008.

MULBERRY, G. et al. Evaluation of a waterless, scrubless chlorhexidine gluconate/ethanol surgical scrub for antimicrobial efficacy. Am J Infect Control, v. 29, n. 6, dec. 2001.

NOBLE, W.C; SOMERVILLE, D.A. Microbiology of human skin. J Clin Pathol, Philadelphia, v.34, n. 9, set. 1981.

OLIVEIRA, A.C.; CIOSAK, S.I. Infecção de sítio cirúrgico em hospital universitário: vigilância pós-alta e fatores de risco. Rev. esc. enferm. USP, São Paulo, v. 41, n. 2, Jun 2007.

ROSENGREN H., CURA C., SMITH S. Uma atualização sobre a profilaxia antibiótica em cirurgia dermatológica. Curr Derm Rep 2012; Volume 1 (2): 55-66

SANTOS, M.L.G.; TEIXEIRA, R.R.; DIOGO-FILHO, A. Surgical site infections in adults patients undergoing of clean and contaminated surgeries at a university Brazilian hospital. Arq. Gastroenterol., São Paulo, v. 47, n. 4, dez. 2010.

SATTAR, S.A; ANSARI, S.A. The fingerpad protocol to assess hygienic hand antiseptics against viruses. J Virol Methods, v. 103, n.2, maio. 2002.

WHO (WORLD HEATH ORGANIZATION). The WHO Guidelines on Hand Hygiene in Health Care. First Global Patient Safety Challenge Clean Care Is Safer Care.Geneva: WHO Press, 2009. 262p. 